\title{
Physical activity levels among Malawian adults living with HIV and receiving anti-retroviral therapy
}

\author{
Enock M. Chisati ${ }^{1,2}$, Francis Munthali3 ${ }^{3}$ Demitri Constantinou ${ }^{4}$, Fanuel Lampiao ${ }^{5,6}$ \\ 1. Department of Physiotherapy, College of Medicine, University of Malawi, Blantyre, Malawi \\ 2. Consortium for Advanced Research Training in Africa (CARTA), Nairobi, Kenya \\ 3. Queen Elizabeth Central Hospital, Blantyre, Malawi \\ 4. Center for Exercise Science and Sports Medicine, FIMS Collaborating Center of Sports Medicine, University of the Witwatersrand, Johannesburg, \\ South Africa \\ 5. Department of Biomedical Sciences, College of Medicine, University of Malawi, Blantyre, Malawi \\ 6. Africa Centre of Excellence in Public Health and Herbal Medicine (ACEPHEM).
}

\section{Introduction}

\section{Abstract}

Despite increasing life expectancy among people living with HIV (PLWHIV), anti-retroviral therapy (ART) side effects, HIV chronic inflammation and co-morbidities may limit functional abilities and reduced participation in exercises and physical activity (PA). PA improves wellbeing and overall quality of life of PLWHIV. In Malawi, there is paucity of information regarding PA levels among Malawians living with HIV and receiving ART. Therefore, this study aimed at determining PA levels among PLWHIV and receiving ART in Malawi.

\section{Methods}

A quantitative cross-sectional design was employed. Eligible participants were male and female adults aged 18-45 years living with HIV receiving ART for at least 1 year. The participants were recruited from Limbe Health Center, Gateway Health Center and Queen Elizabeth Central Hospital (QECH) in Blantyre, Malawi. The International Physical Activity Questionnaire (IPAQ) was used to assess the PA levels. A Stadiometer (HS-DBS00361, Model: 1127154) was used to measure weight (kg) and height (cm) of the participants.

\section{Results}

A total of 213 participants were recruited. There were more females than male participants ( $\mathrm{n}=132 \mathrm{females}$ ). Overall, the mean age of all participants was $37 \pm 6.5$ years and they were within normal body weight $(B M I=23 \pm 4.0)$. Many participants $(\mathrm{n}=85,40 \%)$ had low PA levels followed by those who were moderately physically active ( $\mathrm{n}=75,36 \%$ ). A larger proportion of the female participants $(51 \%)$ had low PA levels compared to males $(22 \%)$. Forty-two percent of participants with 1-3 years of ART had low PA whereas $39 \%$ with $>3$ years ART had low PA.

\section{Conclusion}

Most PLWHIV and receiving ART in the sample have low PA levels. The study has also revealed that proportionally more females than males had low PA levels.

Key Words

Physical activity, anti-retroviral therapy (ART), people living with HIV (PLWHIV)

\section{Introduction}

It is estimated that over 30 million people are infected with human immunodeficiency virus (HIV) with a majority of them living in sub-Saharan Africa ${ }^{1}$. Infection with $\mathrm{HIV}$ is increasingly considered a chronic illness, with the introduction of anti-retroviral therapy (ART) having significantly improved life expectancy among people living with HIV (PLWHIV) ${ }^{2}$. PLWHIV are living longer and aging with comorbidities associated with $\mathrm{HIV}^{3,4}$. Prevalent comorbidities such as cardiovascular diseases, chronic obstructive pulmonary disease, cancers, arthritis, osteoporosis and liver disease develop earlier and more frequently in PLWHIV than in individuals who are not infected with $\mathrm{HIV}^{5,6}$. Side effects of ART such as peripheral neuropathy, osteopaenia, anaemia and fatigue ${ }^{7,8}$ as well as HIV-related chronic inflammation have been implicated as risk factors that increase the incidence of comorbidities ${ }^{9,10}$. Decreases in PA among PLWHIV could be exacerbated by most of the HIV related comorbidities ${ }^{11}$.
PA is defined as any bodily movement produced by skeletal muscles that results in energy expenditure ${ }^{12}$. PA and exercise are as effective as pharmacological interventions in preventing cardiovascular diseases and mortality in the general population ${ }^{13}$. The benefits of regular PA in the general population include controlling body weight, boosting immunity, preventing non-communicable diseases, increasing fitness and improving mental health ${ }^{14}$. PA has been recommended as a therapeutic intervention that can promote health and quality of life for PLWHIV ${ }^{15-20}$. Existing evidence shows that regular PA improves fitness, body composition, muscle strength, psychological wellbeing and quality of life among people living with $\mathrm{HIV}^{18-20}$. PA reduces central fat and metabolic consequences that result from fat accumulation in PLWHIV $^{21}$. Since higher PA levels are associated with many benefits, including lowering cardiovascular disease risk, the leading cause of mortality in people living with $\mathrm{HIV}^{22,23}$, understanding PA levels among people living with HIV and receiving ART is critical to improving long-term health 
outcomes.

The World Health Organization (WHO) guidelines on PA for health recommend aerobic PA of either 150 minutes of moderate intensity or 75 minutes of vigorous intensity per week for adults aged 18-64 years ${ }^{24}$. In addition, WHO recommends muscle strengthening activities involving all major muscle groups that are done 2 or more days per week $^{24}$. Although the benefits of regular PA are enormous, most PLWHIV are insufficiently physically active ${ }^{14,15,25,26}$. Factors such as opportunistic infections, side effects of anti-retroviral drugs, depression and body pain have been implicated as barriers towards the engagement of PLWHIV in regular $\mathrm{PA}^{27}$. PA levels of people living with HIV in Malawi have not been investigated. Therefore, the aim of this study was to determine levels of PA among people living with HIV and receiving ART in Blantyre, Malawi.

\section{Methods}

\section{Study design, study sample and recruitment}

This was a quantitative cross-sectional study consisting of 81 male $(38 \%)$ and 132 female (62\%) adults living with HIV and receiving ART recruited using convenience sampling. Participants were recruited from Limbe Health Centre, Gateway Health Center and Queen Elizabeth Central Hospital (QECH) in Blantyre, Malawi. As the participants came for refilling their ART medication, they were requested to attend a health talk regarding the purpose of the study and requesting their participation. This health talk was conducted by the researchers and the health workers at the clinic. After getting their ART refill, willing participants were directed into a separate room where the aim and objectives of the study were again explained and screening for eligibility was done. Consent was obtained from eligible and willing participants. All ethical procedures were followed and privacy and confidentiality were ensured by allocating codes to the participants. The study was approved by the University of Malawi's College of Medicine Research and Ethics Committee (COMREC) and is in conformity with the laws of Malawi and the Declaration of Helsinki.

\section{Study period}

Data for the study was collected from 28 February 2018 to 30 January, 2019. During this period all eligible people living with HIV and receiving ART from Queen Elizabeth Central Hospital, Limbe Health Center and Gateway Health Center were requested to participate in the study.

\section{Selection criteria}

Male and female adults aged 18-45 years living with HIV who were receiving ART for at least 1 year and were adhering to ART treatment were included. Participants who had been on ART for more than 1 year were selected because ART side effects that may affect PA levels tend to manifest after 1 year ${ }^{28,29}$. Pregnant mothers, subjects hospitalized in the previous 2 weeks and all subjects with well-known neurological, cardiorespiratory, orthopaedic or haematological conditions, opportunistic infections and cancers were excluded from the study since such conditions may affect individuals' participation in PA.

\section{Equipment}

A Stadiometer (HS-DBS00361, Model: 1127154, Supplied by Taida, Blantyre, Malawi) was used to obtain the weight (kg) and height $(\mathrm{cm})$ of subjects. A health passport was used to cross check the day the subject started ART and the default rate. The International Physical Activity Questionnaire (IPAQ) was used to collect data on PA levels of the participants. Guidelines for data processing of the IPAQ were used to categorize data into levels of PA for each participant.

\section{Variables}

\section{Body weight and height}

Body weight $(\mathrm{kg})$ and height measurements were obtained using a Stadiometer (HS-DBS00361, Model: 1127154) following manufacturer guidelines.

\section{Body mass index}

Body mass index (BMI) for each participant was calculated by dividing weight measurement $(\mathrm{kg})$ by the square of the height measurement $\left(\mathrm{m}^{2}\right)$.

\section{Physical activity level}

PA levels were obtained using the self-administered short version of the IPAQ. The IPAQ comprised questions on frequency, intensity and duration of PA that participants do as part of their everyday life in the previous 7 days. Questions were asked such as "During the last 7 days, on how many days did you do vigorous physical activities like heavy lifting, digging, gardening, aerobics, or fast bicycling?"; "How much time did you usually spend doing vigorous physical activities on one of those days?"; "During the last 7 days, on how many days did you do moderate physical activities like carrying light loads, bicycling at a regular pace, or participating in light sporting events?"; "How much time did you usually spend doing moderate physical activities on one of those days?" and so on. To ensure that the questions in the local language (Chichewa) were conceptually equivalent with the English questions in the IPAQ, forward and backward translation was performed by independent translators. The IPAQ was proven to be a valid and reliable tool for measuring PA among adults aged 18-65 in diverse settings ${ }^{30}$.

Variable minutes spent on doing PAs were recorded. The minutes were calculated into metabolic equivalents (METs). METs are defined as multiples of the resting metabolic rate $\left(1 \mathrm{MET}=3.5 \mathrm{ml} \mathrm{O}_{2} \mathrm{~kg}^{-1} \mathrm{~min}^{-1}\right)$ and MET-minutes were calculated by multiplying the MET score of an activity (an equivalence of kilocalories for a $60-\mathrm{kg}$ person) by the minutes performed ${ }^{31}$.

Participants were categorized into low, moderate and high PA levels. Low level PA comprised participants having the lowest PA and did not meet the criteria for moderate or high PA levels. Moderate level PA comprised participants who did 3 or more days of vigorous PA for at least 20 minutes per day. Similarly, participants who performed 5 or more days of walking or moderate intensity PA for at least 30 minutes per day, fell into the moderate-level PA category. High level PA comprised participants who performed at least 3 days of vigorous intensity PA accumulating at least 1500 MET minutes per week. Similarly, participants who performed 7 or more days of any combination of vigorous PA, moderate intensity PA or walking achieving a total of at least 3000 MET minutes per week fell into the high-level PA category.

\section{Duration on anti-retroviral therapy}

The period on ART was obtained through asking the participants and cross-checking in the health passport. Participants were included if the period they have been 
receiving ART was 1 year or more. The duration was divided into categories of $1-3$ years and above 3 years because side effects of ART that could affect PA become more pronounced after 3 years ${ }^{28,29}$.

\section{Data analysis}

Data was entered into Microsoft Excel 2016 after which METs for each participant's PA level were computed using guidelines for data processing of the IPAQ ${ }^{31}$. Participants were categorized into low PA, moderate PA and high PA levels. Statistical Package for the Social Science (IBM SPSS statistics for Windows, Version 20.0. Armonk, New York) was used to analyse the data. Descriptive statistics with mean and standard deviation were used to characterize the data variables. All data assumed normal distribution.

\section{Results}

The study investigated a sample of 213 adult Malawians aged between 18 and 45 living with HIV and receiving ART from Limbe Health Center, Gateway Health Center and QECH in Blantyre. Overall, the mean age of all participants

Table 1. Baseline characteristics of participants

\begin{tabular}{llll}
\hline & Male & Female & Total \\
& $\mathrm{n}=81$ & $\mathrm{n}=132$ & $\mathrm{n}=213$ \\
\hline Age (years) & $37 \pm 6.7$ & $36 \pm 6.3$ & $37 \pm 6.5$ \\
Weight $(\mathrm{kg})$ & $59 \pm 8.9$ & $59 \pm 10.5$ & $59 \pm 9.9$ \\
Height $(\mathrm{cm})$ & $164 \pm 0.8$ & $158 \pm 0.8$ & $160 \pm 0.8$ \\
BMI $\left(\mathrm{kg} / \mathrm{m}^{2}\right)$ & $22 \pm 3.1$ & $24 \pm 4.4$ & $23 \pm 4.0$ \\
\hline
\end{tabular}

All data are in mean \pm standard deviation.

BMI, body mass index.

Table 2. Proportions of PA levels of participants by ART duration

\begin{tabular}{lcc}
\hline & ART duration & \\
& $1-3$ years & $>3$ years \\
\hline Low PA & $32(42 \%)$ & $53(39 \%)$ \\
Moderate PA & $25(32 \%)$ & $51(38 \%)$ \\
High PA & $20(26 \%)$ & $32(23 \%)$ \\
\hline Total & $77(100 \%)$ & $136(100 \%)$ \\
\hline
\end{tabular}

All data are $n(\%)$.

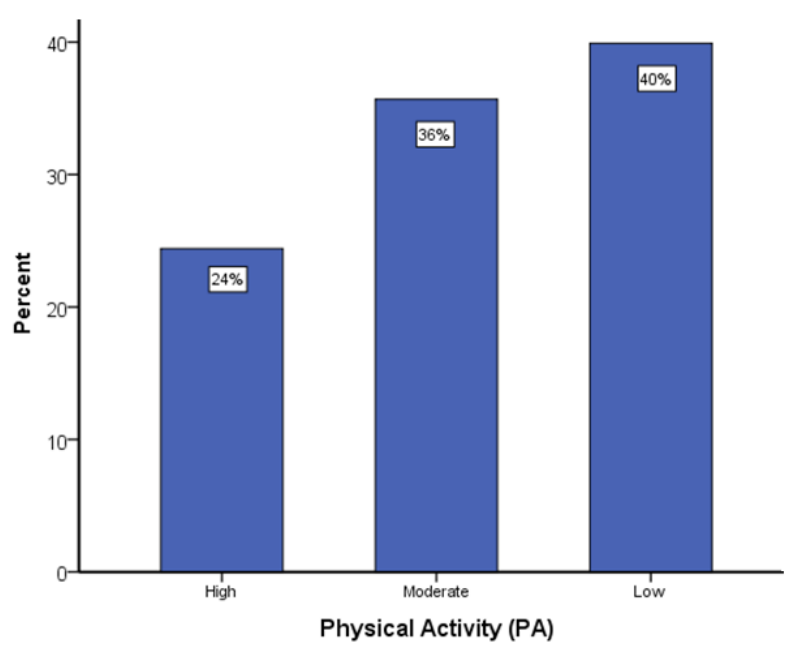

Figure 1: Physical activity (PA) levels of all participants. was $37 \pm 6.5$ years and they were within normal body weight $(\mathrm{BMI}=23 \pm 4.0)$. There were more female than male participants ( $n=132$ female) with mean age of $36 \pm 6.3$ years. Baseline characteristics of participants are presented in Table 1. Most participants (40\%) had low PA levels, followed by those who were moderately physically active $(36 \%)$ while a smaller number of participants $(24 \%)$ had high PA levels (Figure 1). A larger number of females $(51 \%)$ had low PA levels compared to males (22\%) (Figure 2).

Out of all 77 participants with 1-3 years of ART, $32(42 \%)$ had low PA levels and $53(39 \%)$ out of 136 participants with $>3$ years ART had low PA levels (Table 2).

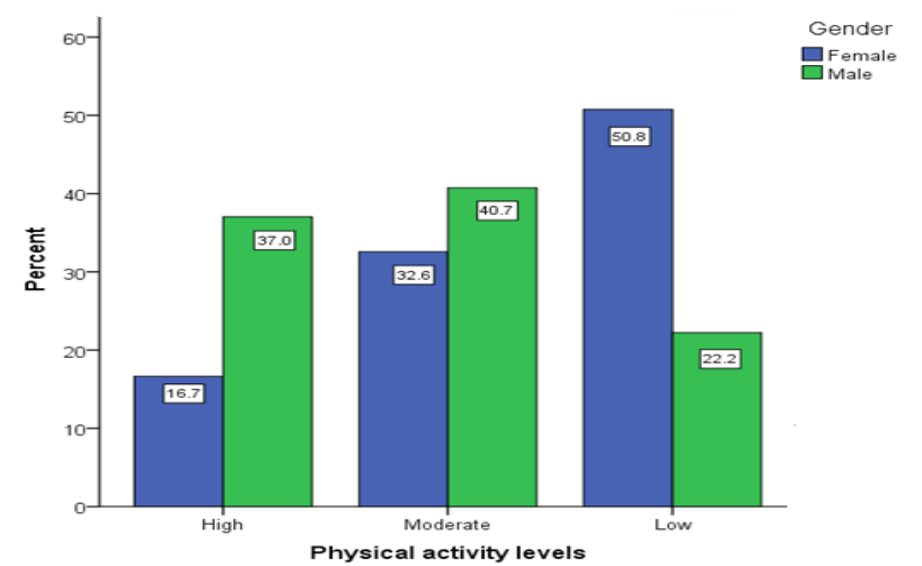

Figure 2: Physical activity (PA) levels of all participants stratified by gender.

\section{Discussion}

The study was conducted to determine the levels of PA in a sample of people living with HIV in Blantyre, Malawi. Contrary to WHO recommendations for $\mathrm{PA}^{24}$, current findings show that a considerable proportion of PLWHIV and receiving ART in Blantyre, Malawi are insufficiently physically active. Our results are consistent with other studies that have shown low levels of PA among people living with HIV and receiving ART ${ }^{14,15,26,32,33}$. Other reports have suggested that lower levels of PA among PLWHIV could be due to lower educational levels, presence of lipodystrophy, body pains, depression and opportunistic infections ${ }^{27}$. It should be noted that some of the indicated factors leading to reduced levels of PA among people living with HIV may be interrelated. For example, presence of lipodystrophy which contributes to reduced PA levels of PLWHIV is a known side effect of stavudine-based ART, which the WHO recommended to be phased out ${ }^{34}$. In our study, none of the participants was taking stavudine-based ART as it was phased out in $2011^{35}$. In addition, those with opportunistic infections were excluded from the study. Therefore, the presence of lipodystrophy and opportunistic infection may not explain the lower PA levels in our study. Low PA levels among participants in our study could also be due to body pains and depression because these are common among PLWHIV and receiving ART ${ }^{27}$. However, this suggestion should be considered with caution since our study did not investigate depression and body pain among the participants. Although older age has also been suggested as leading to low PA among PLWHIV ${ }^{27}$, participants in the current study were young adults aged between 18 and 45 years. Frantz et al. also reported lower PA levels among a majority of the younger population (15-34 years) of PLWHIV ${ }^{32}$. This is a cause for concern because PA levels tend to decrease with 
aging ${ }^{36,37}$. Younger adults are considered a productive age group in society, hence having low PA levels poses a public health challenge as they will be unable to participate in daily activities and contribute to the economy of the country.

Results of the study also show that a larger number of female participants had low PA levels compared to male participants. Our results are consistent with a number of studies reporting that males are more active than females ${ }^{38-40}$. Higher PA levels among Malawian males could be expected since a large percentage of the male population is involved in manual labour and agricultural operations that demand considerable physical capacity. Lifestyle activities such as walking long distances to work places, industrial activities that involve pushing levers, land preparation and post-harvest processing of farm produce may also increase physical capacity among Malawian males.

Our results also show a similar proportion of participants with low PA levels between those with 1-3 years ART and those with over 3 years ART. Our findings are contrary to results from a study by Frantz et al., which reported lower levels of PA in participants who were on ART for more than 4 years in Rwanda ${ }^{32}$. This could be due to differences in the age range of the study participants. The study done in Rwanda included older participants (age ranged from 18 to 75) while participants in the current study were younger adults aged between 18 to 45 . As revealed by evidence from other studies, lower levels of PA are associated with older $\operatorname{age}^{27}$.

\section{Limitations}

The study focused on the active age groups of people living with HIV in Malawi population other than both age extremities. In addition, the current study used a subjective approach of obtaining data through self-reported questionnaire that may result in reporting bias. The study was conducted among urban settlers living within Blantyre who may have different PAs compared to rural settlers. Hence there is need for more studies to be conducted in other rural areas around Malawi.

\section{Conclusion}

This study has highlighted that a larger number of people living with HIV and receiving ART within Blantyre, Malawi have low PA levels, with an almost similar percentage being moderately physically active. Furthermore, a larger number of females have low PA levels compared to males.

\section{Authors' contributions}

EC made substantial contributions to the conception, design of the work and drafted the work. FM collected and assisted in analysing data for the study while DC and FL revised the work critically for important intellectual content. All authors are in agreement to be accountable for all aspects of the work in ensuring that questions related to the accuracy or integrity of any part of the work are appropriately investigated and resolved.

\section{Data availability statement}

Data for the study cannot be shared publicly because the data contains potentially identifying information. The restriction has been imposed by the College of Medicine
Ethics Committee (COMREC), an IRB that approved the study. Data are available from COMREC (Email: comrec@) medcol.mw) for researchers who meet the criteria for access to confidential data.

\section{Funding}

This research was supported by the Consortium for Advanced Research Training in Africa (CARTA). CARTA is jointly led by the African Population and Health Research Center and the University of the Witwatersrand and funded by the Carnegie Corporation of New York (Grant No. B 8606.R02), Sida (Grant No. 54100113), the DELTAS Africa Initiative (Grant No. 107768/Z/15/Z) and Deutscher Akademischer Austauschdienst (DAAD). The DELTAS Africa Initiative is an independent funding scheme of the Alliance for Accelerating Excellence in Science in Africa (AESA) of the African Academy of Sciences (AAS) and supported by the Development Planning and Coordinating Agency (NEPAD Agency) of the New Partnership for Africa, with funding from the Wellcome Trust (UK) and the UK government. The statements made and views expressed are solely the responsibility of the Fellow. Enock Chisati is a cohort 7 CARTA PhD fellow.

\section{Competing interests}

The authors state no conflicts of interest in this research or for the development of the article.

\section{References}

1. World Health Organization. Global AIDS Update 2016. Geneva: WHO; 2016.

2. Murray CJL, Vos T, Lozano R, Naghavi M, Flaxman AD, Michaud $\mathrm{C}$, et al. Disability-adjusted life years (DALYs) for 291 diseases and injuries in 21 regions, 1990-2010: a systematic analysis for the Global Burden of Disease Study 2010. Lancet. 2012;380(9859):2197-223. doi:http://dx.doi.org/10.1016/S0140-6736(12)61689-4.

3. Morris A, Gingo MR, George MP, Lucht L, Kessinger C, Hillenbrand $\mathrm{M}$, et al. Cardiopulmonary function in individuals with HIV infection in the antiretroviral therapy era. AIDS. 2012;26(6):731-40. doi: 10.1097/ QAD.0b013e32835099ae.Cardiopulmonary.

4. Nlooto M. Comorbidities of HIV infection and health care seeking behavior among HIV infected patients attending public sector healthcare facilities in KwaZulu-Natal: A cross sectional study. PLoS One. 2017;12(2):1-15. doi:10.1371/journal.pone.0170983.

5. Deeks SG, Phillips AN. HIV infection, antiretroviral treatment, ageing, and non-AIDS related morbidity. BMJ. 2009;338:288-292. doi:10.1136/bmj.a3172.

6. Schouten J, Wit FW, Stolte IG, Kootstra NA, Valk MV, Geerlings SE. Cross-sectional comparison of the prevalence of age-associated comorbidities and their risk factors between HIV-infected and uninfected individuals: The AGEhIV cohort study. Clin Infect Dis. 2014;59(12):1787-97. doi:10.1093/cid/ciu701.

7. Khan K, Khan AH, Sulaiman SA, Soo CT, Ahsan R. Adverse effect of highly active anti-retroviral therapy (HAART) in HIV/AIDS patients. Indian J Pharm Pract. 2014;7(3):6-11. doi:10.5530/ijopp.7.3.7.

8. Alonge T, Okoje-Adesomoju V, Atalabi O, Obamuyide H, Olaleye D, Adewole I. Prevalence of abnormal bone mineral density in HIV-positive patients in Ibadan, Nigeria. J West African Coll Surg. 2013;3(4):1-14.

9. Dillon DG, Gurdasani D, Riha J, Ekoru K, Asiki G, Mayanja BN, et al. Association of HIV and ART with cardiometabolic traits in subSaharan Africa: A systematic review and meta-analysis. Int J Epidemiol. 2013;42(6):1754-71. doi:10.1093/ije/dyt198.

10. Hearps AC, Martin GE, Rajasuriar R, Crowe SM. Inflammatory https://doi.org/10.4314/mmj.v32i1.3: 
co-morbidities in HIV+ individuals: learning lessons from healthy ageing. Curr HIV/AIDS Rep. 2014;11(1):20-34. doi:10.1007/s11904013-0190-8.

11. Baranoski AS, Harris A, Michaels D, Miciek R, Storer T, Sebastiani $\mathrm{P}$, et al. Relationship between poor physical function, inflammatory markers and comorbidities in HIV-infected women on antiretroviral therapy. J Women's Health. 2014;23(1):69-76. doi:10.1089/ jwh.2013.4367.

12. Caspersen CJ, Powell KE, Christenson GM. Physical activity, exercise, and physical fitness: definitions and distinctions for healthrelated research. Public Health Rep. 1985;100(2):126-31.

13. Naci H, Ioannidis JPA. Comparative effectiveness of exercise and drug interventions on mortality outcomes : metaepidemiological study. BMJ. 2013;347:1-14. doi:10.1136/bmj.f5577.

14. Vancampfort D, Mugisha J, Hert M De, Probst M, Firth J, Gorczynski $\mathrm{P}$, et al. Global physical activity levels among people living with HIV : a systematic review and meta-analysis. Disabil Rehabil. 2017;40(4):38897. doi:10.1080/09638288.2016.1260645..

15. Gomes Neto M, Conceição CS, Ogalha C, Brites C. Aerobic capacity and health-related quality of life in adults HIV-infected patients with and without lipodystrophy. Braz J Infect Dis. 2015;20(1):76-80. doi:10.1016/j.bjid.2015.11.001.

16. Gomes-Neto M, Conceição CS, Oliveira Carvalho V, Brites C. A systematic review of the effects of different types of therapeutic exercise on physiologic and functional measurements in patients with HIV/ AIDS. Clinics. 2013;68(8):1157-67. doi:10.6061/clinics/2013(08)16.

17. Leach LL, Bassett SH, Smithdorf G, Andrews BS, Travill AL. A systematic review of the effects of exercise interventions on body composition in HIV+ adults. Open AIDS J. 2015;9:66-79. doi:10.2174 $/ 1874613601509010066$

18. Neto MG, Conceico CS, Carvalho VO, Brites C. Effects of combined aerobic and resistance exercise on exercise capacity, muscle strength and quality of life in HIV-infected patients: A systematic review and meta-analysis. PLoS One. 2015;10(9):e0138066. doi:10.1371/journal. pone. 0138066 .

19. O’Brien KK, Tynan A-M, Nixon SA, Glazier RH. Effectiveness of aerobic exercise for adults living with HIV: systematic review and meta- analysis using the Cochrane Collaboration Protocol. BMC Infect Dis. 2016;16:182. doi:10.1186/s12879-016-1478-2.

20. O'Brien K, Tynan A-M, Nixon S, Glazier RH. Effects of progressive resistive exercise in adults living with HIV/AIDS: systematic review and meta-analysis of randomized trials. AIDS Care. 2008;20(6):63153. doi:10.1080/09540120701661708.

21. Segatto AM, Junior F, Dos Santos V, Cristina K, Alves P, Barbosa DA, et al. Lipodystrophy in HIV/AIDS patients with different levels of physical activity while on antiretroviral therapy. Rev Soc Bras Med Trop. 2011;44(4):420-4.

22. Dirajlal-Fargo S, Webel AR, Longenecker CT, Kinley B, Labbato D, Sattar A, et al. The effect of physical activity on cardiometabolic health and inflammation in treated HIV infection. Antivir Ther 2016;21(3):237-45. doi:10.3851/IMP2998.

23. Farahani M, Mulinder H, Farahani A, Marlink R. Prevalence and distribution of non-AIDS causes of death among HIV-infected individuals receiving antiretroviral therapy: a systematic review and meta-analysis. Int J STD AIDS. 2017;28(7):636-50. doi:10.1177/0956462416632428.

24. World Health Organization. Global Recommendations on Physical Activity for Health. Geneva: WHO; 2010.

25. Chisati EM, Vasseljen O. Aerobic endurance in HIV-positive young adults and HIV-negative controls in Malawi. Malawi Med J.
2015;27(1):5-9. doi:10.4314/mmj.v27i1.2.

26. Fillipas S, Bowtell-Harris CA, Oldmeadow LB, Cicuttini F, Holland AE, Cherry CL. Physical activity uptake in patients with HIV: who does how much? Int J STD AIDS. 2008;19:514-8.

27. Vancampfort D, Mugisha J, Richards J, De Hert M, Probst M, Stubbs B. Physical activity correlates in people living with HIV/AIDS : a systematic review of 45 studies. Disabil Rehabil. 2018;40(14):161829. doi:10.1080/09638288.2017.1306587.

28. Evans SR, Elias RJ, Chen H, Yeh T, Lee AJ, Schifitto G, et al. Peripheral neuropathy in HIV : prevalence and risk factors. AIDS 2012;25(7):919-28. doi:10.1097/QAD.0b013e328345889d.Peripheral.

29. Duvivier C, Kolta S, Assoumou L, Ghosn J, Rozenberg S, Murphy $\mathrm{R}$, et al. Greater decrease in bone mineral density with protease inhibitor regimens compared with nonnucleoside reverse transcriptase inhibitor regimens in HIV-1 infected naive patients. AIDS. 2009;23(7):817-24. doi:10.1097/QAD.0b013e328328f789.

30. Bassett DR. International physical activity questionnaire: 12-country reliability and validity. Med Sci Sports Exerc. 2003;35(8):1396. doi:10.1249/01.MSS.0000078923.96621.1D.

31. IPAQ Research Committee. Guidelines for Data Processing and Analysis of the International Physical Activity Questionnaire (IPAQ) - Short and Long Forms. IPAQ; 2005. Available from www.ipaq.ki.se [cited 2019 March 20]

32. Frantz JM, Murenzi A. The physical activity levels among people living with human immunodeficiency virus/acquired immunodeficiency syndrome receiving high active antiretroviral therapy in Rwanda. J Soc Asp HIV/AIDS. 2014;10(3-4):113-8. doi:10.1080/17290376.2014.88 6081.

33. Smit E, Crespo CJ, Semba RD, Jaworowicz D, Vlahov D, Ricketts EP, et al. Physical activity in a cohort of HIV-positive and HIV-negative injection drug users. AIDS Care. 2006;18(8):1040-5. doi:10.1080/09540120600580926.

34. World Health Organization. Antiretroviral Therapy for HIV Infection in Adults and Adolescents: Recommendations for a Public Health Approach-2010 Revision. Geneva: WHO; 2010.

35. Malawi Government. Global AIDS Response Progress Report (GARPR): Malawi Progress Report for 2013. Malawi Government; 2014. https://www.google.com/search?client=firefox-b-d\&q=Malawi ++Government.+Global+AIDS+Response+Progress+Report+\%28G ARPR $\% 29 \% 3 \mathrm{~A}+$ Malawi+Progress+Report+for+2013.+Malawi+Gov ernment $\% 3 \mathrm{~B}+2014$.

36. Milanovic Z, Pantelic S, Trajkovic N, Sporis G, Kostic R, James N. Age-related decrease in physical activity and functional fitness among elderly men and women. Clin Interv Aging. 2013;8:549-56.

37. Sagatun Å, Kolle E, Anderssen SA, Thoresen M, Søgaard AJ. Three-year follow-up of physical activity in Norwegian youth from two ethnic groups: associations with socio-demographic factors. BMC Public Health. 2008;8:419.:1-9. doi:10.1186/1471-2458-8-419.

38. Azevedo MR, Luiza C, Araújo P, Reichert FF, Siqueira FV, Cozzensa $\mathrm{M}$, et al. Gender differences in leisure-time physical activity. Int J Public Health. 2007;52(2007):8-15. doi:10.1007/s00038-006-5062-1.

39. Martinez-Gonzalez MA, Varo JJ, Santos JL, Irala JDE, Gibney M, Kearney J, et al. Prevalence of physical activity during leisure time in the European Union. Med Sci Sport Exerc. 2001;33(7):1142-6.

40. Monteiro CA, Conde WL, Matsudo SM, Matsudo VR, Bonseñor IM, Lotufo PA. A descriptive epidemiology of leisure-time physical activity in Brazil, 1996-1997. Pan Am J Public Health. 2003;14(4):246-54. 